\title{
PASH Syndrome: Comparing Pathogenic Mechanism of Pyoderma Gangrenosum, Acne and Suppurative Hidradenitis
}

\author{
Roxana Ioana Nedelcu ${ }^{1,2 *}$, Gabriela Turcu ${ }^{1,2,3 *}$, Mihaela Balaban ${ }^{1,2}$, Anastasia Hodorogea ${ }^{1,3}$, Mi- \\ haela Antohe ${ }^{1,2}$, Andreea Calinescu, ${ }^{1,2}$, Laurentiu Stratan ${ }^{1,4}$, Mirela Cioplea ${ }^{1,3}$, Nickolai Nayde- \\ nov $^{5}$, Aliona Sineva ${ }^{6}$, Raluca Popescu ${ }^{1,3}$, Catalin Mihai Popescu ${ }^{1,3}$, Ionela Hulea ${ }^{1}$, Daniela Adria- \\ na Ion ${ }^{1}$, Sabina Andrada Zurac ${ }^{1,3}$ and Alice Brinzea ${ }^{1,3,4 *}$ \\ ${ }^{1}$ Carol Davila University of Medicine and Pharmacy, 050474 Bucharest, Romania \\ ${ }^{2}$ Derma $360^{\circ}$ Clinic, 011273 Bucharest, Romania
}

${ }^{3}$ Colentina Clinical Hospital, 020125 Bucharest, Romania

${ }^{4}$ National Institute for Infectious Diseases, 021105 Bucharest, Romania

${ }^{5}$ Dermatology Clinic, Sofia, Bulgaria

${ }^{6}$ Derm AS Clinique, Ruse, Bulgaria

*Corresponding author: Roxana Ioana Nedelcu, Carol Davila University of Medicine and Pharmacy, Derma $360^{\circ} \mathrm{Clinic} 050474$ Bucharest, Romania

To Cite This Article: Roxana Ioana Nedelcu, Gabriela Turcu, Mihaela Balaban, Anastasia Hodorogea, PASH Syndrome: Comparing Pathogenic Mechanism of Pyoderma Gangrenosum, Acne and Suppurative Hidradenitis. 2020 - 11(1). AJBSR.MS.ID.001587. DOI: 10.34297/AJBSR.2020.11.001587.

Received: 眥 November 18, 2020; Published: 眥 November 30, 2020

\begin{abstract}
PASH syndrome is a recently described autoinflammatory disease, consisting of pyoderma gangrenosum (PG), acne and hidradenitis suppurativa (HS). We report a new case of PASH syndrome, highlighting the features of the rare condition. For a better understanding of each of the three diseases and of the PASH syndrome, we reviewed the common and the particular aspects of these pathologies, looking from different angles of inflammation mechanisms. Among the approached topics, we discussed about: increased production of interleukin-1 (IL1) and of other cytokines, altered matrix metalloproteinases expression, multiple roles of neutrophils, genetic profiling and inflammatory infiltrates. As far as we know it is the first time we note the similarities and differences of pathways beyond the neutrophils mediated cutaneous manifestations present in PASH, PG, HS and acne. Our findings emphasize that PASH syndrome may be an inspiring lesson for rethinking autoinflammatory, neutrophilic, innate immune skin diseases.
\end{abstract}

\section{Introduction}

PASH syndrome is a neutrophil mediated inflammatory syndrome, with recurrences and relapses, whose management represents a real challenge [1]. The acronym PASH stands for pyoderma gangrenosum (PG), severe acne (A) and suppurative hidradenitis (SH) [2,3]. This entity was first described in 2012 by Braun-Falco et al, who observed a different association of diseases than the one previously known as PAPA syndrome (pyogenic sterile arthritis, pyoderma gangrenosum and severe acne) [2]. A gathering of pyogenic sterile arthritis, pyoderma gangrenosum, acne and hidradenitis suppurativa is known as PAPASH syndrome $[3,4]$ whilst the term "PsaPASH syndrome" was proposed for describing the association of psoriatic arthritis and PASH syndrome [1].

Pyoderma gangrenosum (PG) is a neutrophilic dermatosis, clinically characterized by pustules located especially over the 
lower extremities, which soon become painful erythematous or violaceous ulcerations, with undermined border. It can accompany a hematologic malignancy or a chronic intestinal disease. Pathology report may reveal findings that are not specific: the dermis contains neutrophilic infiltrates (which may be arranged in abscesses or around perivascular lymphocytic infiltrates) and edema. Other disorders, such as bacterial infections (including Mycobacteria), insect bites, venous ulcers, vasculitic processes, self-induced lesions, neoplasm must be ruled out [5].

Acne, a skin disorder affecting the pilosebaceous unit, manifests clinically as papules, pustules, open and closed comedones. Acne is the most common inflammatory dermatosis in the world [6]. Sometimes, it can take a more severe form, which associates nodules, cyst, sinus tracts, accesses and which tends to scar. Syndromic cases of severe acne had been reported PASH/ PAPASH/ SAPHO (synovitis, acne, pustulosis, hyperostosis, osteitis) and

CAH (congenital adrenal hyperplasia) [7].

Suppurative Hidradenitis (SH) is a recurrent chronic inflammatory condition of follicular hair follicles from areas with apocrine glands, most commonly the axillary, inguinal and anogenital region. Clinical lesions can be found in intertriginous areas as suppurative nodules, abscesses, draining sinuses which heal with extensive scars [8-9]. Harley classification consisting of
3 different stages according to the severity of the lesions is used in assessing a patient with hidradenitis suppurativa. It can appear isolated or in the context of various syndromes (PASH/ PAPASH/ follicular occlusion syndrome, etc.) [9]. It was observed that HS in the context of an auto inflammatory syndrome has a poor response to treatment and a recalcitrant behavior [9]. We here present a new case of PASH syndrome and review by comparison the three mentioned multifactorial entities that define the hereditary autoinflammatory syndrome.

\section{Case}

We report the case of a 37-year-old patient, who presented for a pustule located over the left leg, evolving for two days. He was diagnosed with hidradenitis suppurativa since 2009 and acne vulgaris since adolescence. Clinical examination revealed an erythematous and violaceous pustule surrounded by erythema on the leg (Figure 1a) and a few active lesions of acne, accompanied by many atrophic scars on the face (Figure 1b) and cervical and occipital regions. In the axillary region there were hypertrophic scars, multiple sinuses tracts evacuating a purulent content in small amounts, corresponding to Hurley III stage for HS (Figure 1c). He denied any symptoms related to arthritis or inflammatory bowel disease. He admitted having a brother with non-investigated chronic ulcerations on the lower legs.

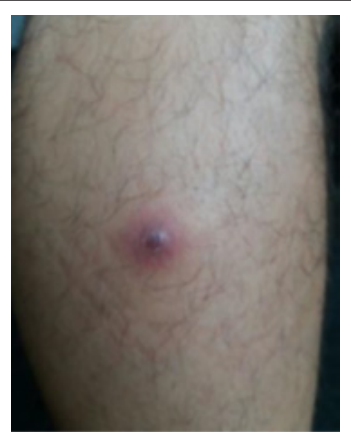

Figure 1a: First pustule of pyoderma gangrenosum

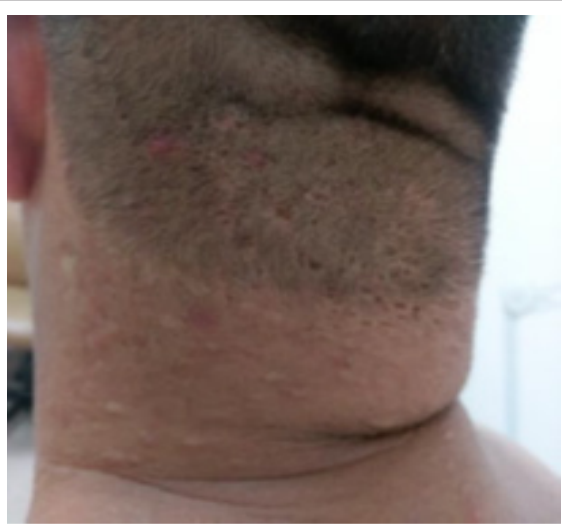

Figure 1b: Acne scars after severe clinical form. 


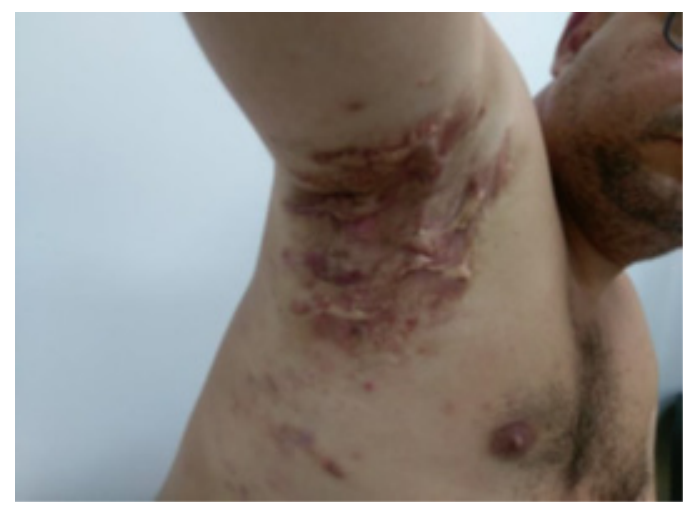

Figure 1c: Hidradenitis suppurativa Harley III stage.

He was initially prescribed a systemic treatment with clindamycin (300 mg twice/daily) and rifampicin (600 mg/daily), but the pustule over the left leg evolved into a painful abscess surrounded by erythema (Figure 1d-e). Soft tissue ultrasound was suggestive of cellulitis and the patient was referred to the
Surgery Department, where he underwent incision and drainage of the abscess, but no purulent material was evacuated (Figure 1f). A Doppler ultrasound examination excluded a deep venous thrombosis. Blood tests were within normal values

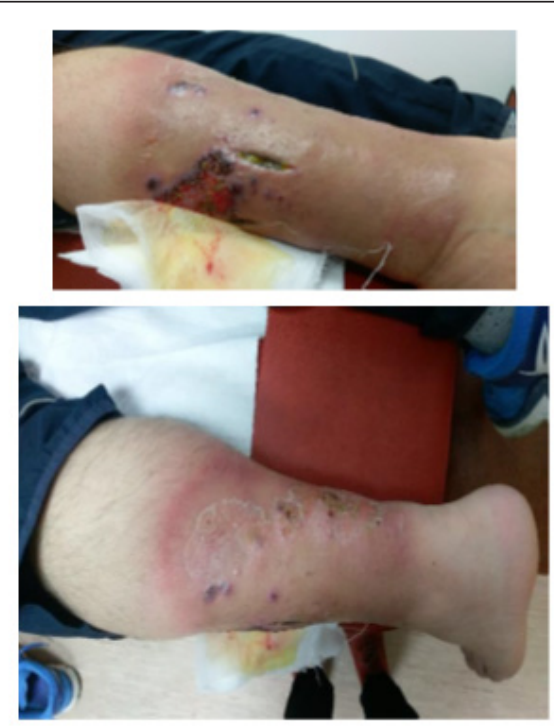

Figure 1d-1e. Clinical aspect of evolving pyoderma gangrenosum.

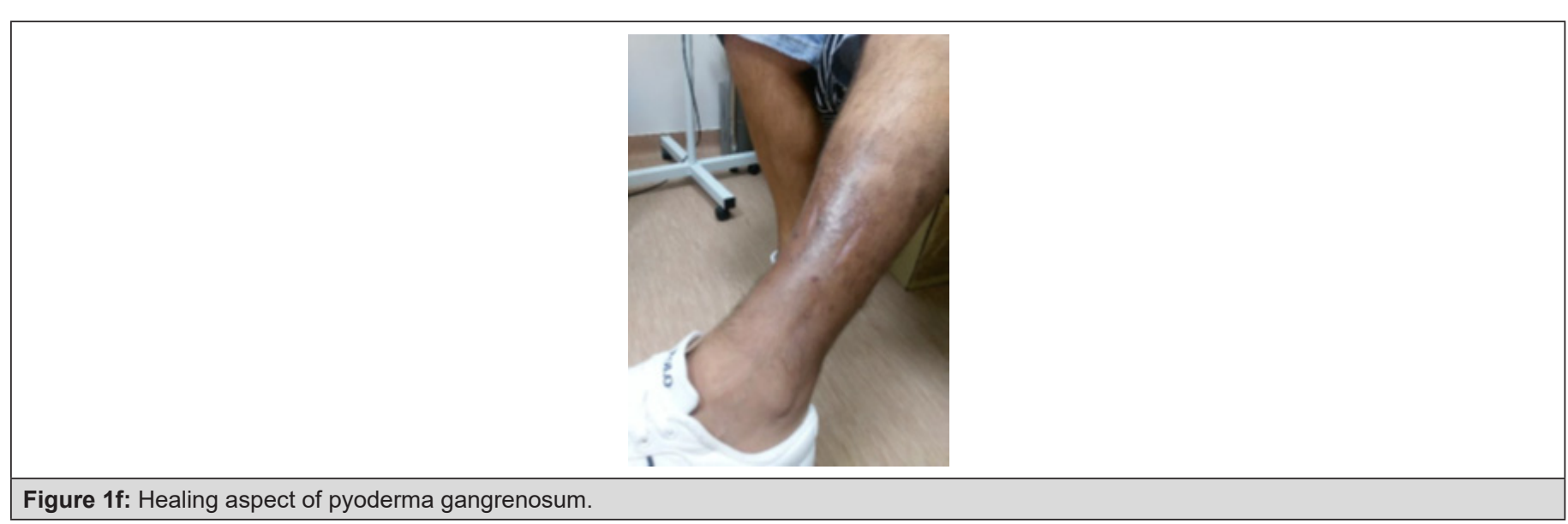


except for inflammatory syndrome, dyslipidemia and mild normocytic normochromic anemia.

Body temperature remained within normal ranges throughout the period. He was advised to stop the previous antibiotic treatment and he was prescribed amoxicillin-clavulanate (1 g every 12 hours) and cyprofloxacyn (500 mg every 12 hours). After 7 days of antibiotic therapy, he returned to our clinic, with the abscess being replaced by a deep and painful ulcer, with undermined, violaceous borders, very suggestive of pyoderma gangrenosum.
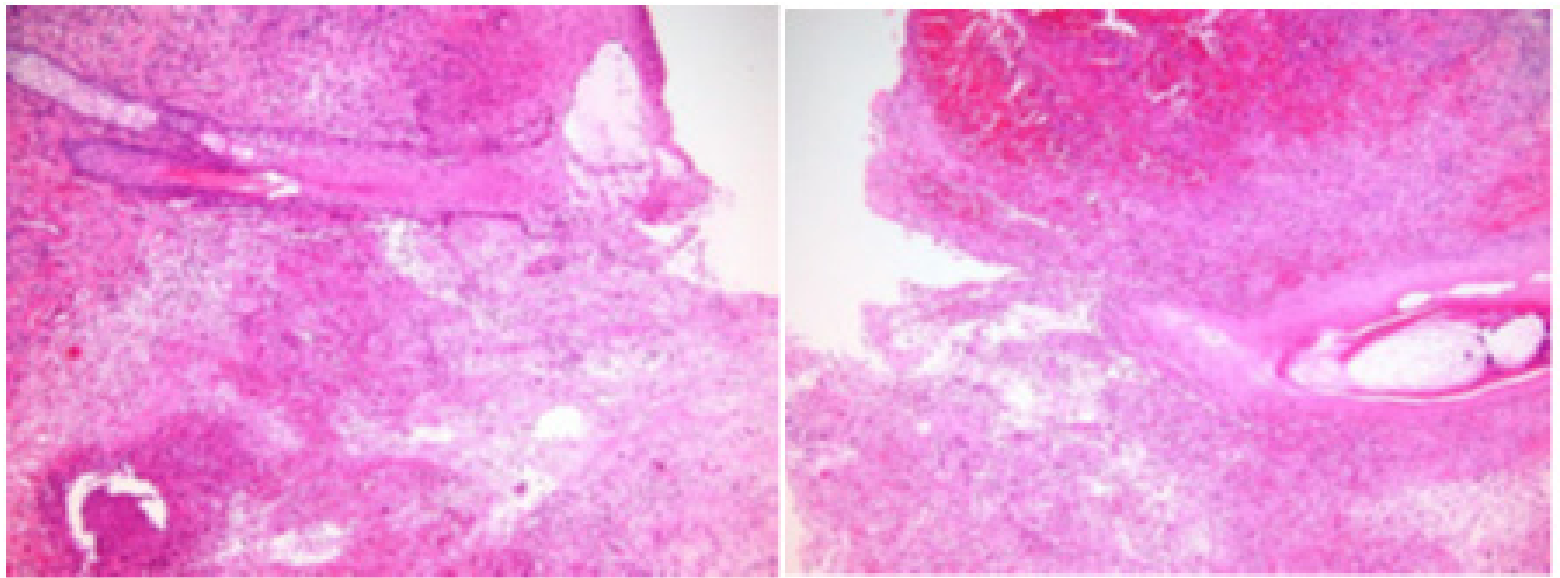

Figure 2a-2b: Ulcerative process with deep, dense folliculocentric neutrophilic inflammatory infiltrate with folliculodestructive features and some leukocytoclasis. HE x 100.

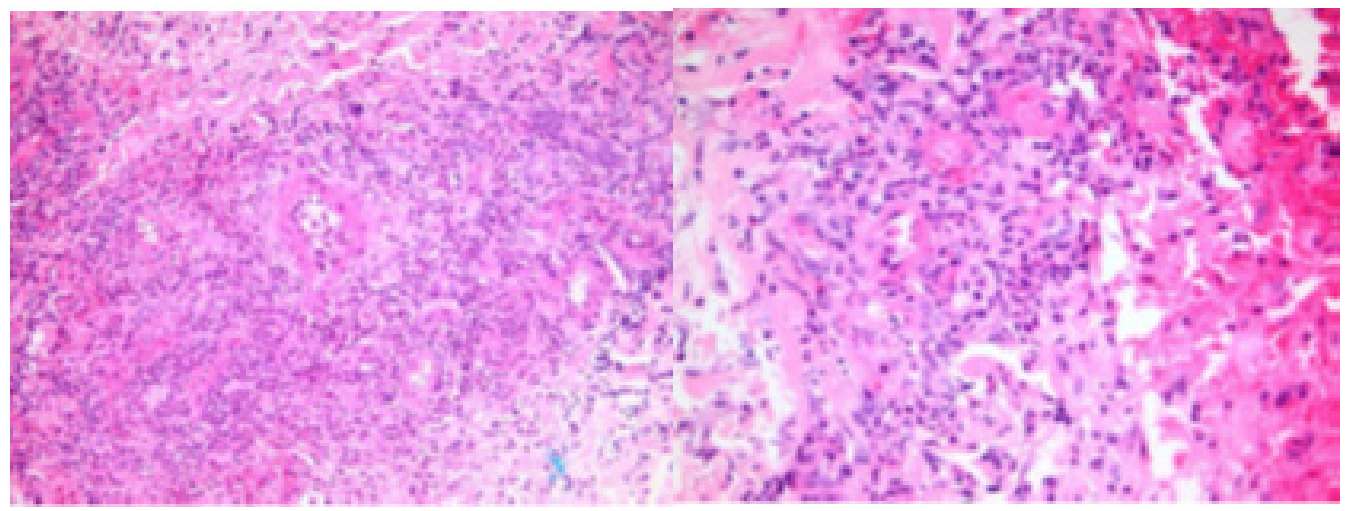

Figure 2c-2d: Secondary vasculitis (intramural fibrin \pm fibrinoid necrosis, intramural neutrophils and intraluminal microthrombi). 3. HE x 200. 4. HE X 400.

A histopathological examination from the ulceration over the left leg revealed polymorphous inflammatory infiltrate with great number of neutrophils, lymphocytes, plasma cell, macrophages, with focal suppuration and small vessels with fibrinoid necrosis and fibrinoid thrombi, findings that were consistent with pyoderma gangrenosum (figure 2a-2d). Considering the association of pyoderma gangrenosum, hidradenitis suppurative and acne in our patient, the final diagnosis was PASH syndrome.

He was prescribed a combination of systemic prednisone $(0.8 \mathrm{~g} /$ $\mathrm{kg}$ and salazopyrin $2 \mathrm{~g}$ /daily) and local therapy (topical steroid and antibiotic, emollient and daily changing of the dressing) with a favorable evolution. Prednisone was slowly tapered over the next months, until a maintenance dose of $0.5 \mathrm{mg} / \mathrm{kg}, 50 \mathrm{mg} /$ daily, with the salazopyrin dose unchanged. At 5-month follow up visit, the ulceration over the left leg was completely healed without any new lesions (figure 1f), HS remained stable and less suppurative, with fewer active acne lesions over the occipitocervical area compared to the first presentation. At 10-month follow up visit, the patient was taking prednisone (20 mg/daily) and salazopyrin (2g/daily) with an unchanged evolution.

\section{Discussion}

Each of the conditions that define PASH syndrome has a relevant psychosocial impact, affecting quality of life, being associated with increased risk for social isolation, anxiety, depression, to suicidal ideation [6].For a better understanding of each of the three diseases and of the PASH syndrome, we aimed to highlight the common and 
particular elements of these pathologies, looking from different angles. Analyzing the literature, we did not identify another study having a similar purpose.

\section{Pathophysiology - inflammation is the key?}

Acne and suppurative hidradenitis are adnexal diseases, both of which involve multifactorial damage of the pilosebaceous unit, while pyoderma gangrenosum involves a vascular neutrophilic reaction that may be folliculocentric. Polymorphous clinically, the classic aspects of these conditions are often characterized by the presence of pustules, inflammatory nodules, exudation, with consequences to disfigurement and functional impotence. Interesting, none of the inflammatory lesion has an infectious trigger as primary stimulus. Hyperactivity of the innate immune system associated with increased IL-1 production and cutaneous inflammation rich in neutrophils of a sterile type not stimulated by a stimulus are characteristics of both autoinflammatory syndromes and of some of the neutrophilic dermatoses. These observations have led to the suggestion that autoinflammatory syndromes and neutrophilic diseases may be part of a broader spectrum of innate immune disorders [10].

Neutrophils are essential inflammatory cells. In the presence of inflammatory agents, neutrophils migrate to the site of the injury and are activated, contributing to the degradation of the triggering factor of the inflammatory process. Accumulation and inappropriate activation of neutrophils, in the absence of an identifiable triggering factor, causes injuries to apparently normal tissues. The predominantly neutrophilic inflammatory response is associated with soreness and pustules in acne. A response of $\mathrm{T}$ helper lymphocytes, giant cells of foreign bodies and neutrophils is associated with the production of inflammatory papules, nodules and cysts.

Impaired immune responses - tissue and circulatory evidence Defining pathophysiological elements for acne are follicular hyperkeratinization, sebum altered production and inflammation [11]. In classical vision, acne is explained by the following events: increased activity of sebaceous glands and hyperseborrhea; abnormal follicular differentiation associated with hyperkeratinization; microbial colonization of the follicular canal followed by innate immune system activation and inflammation [6]. There is still a controversy over the temporal sequence between follicular hyperkeratinization and the onset of inflammation in acne [12]. Further studies have shown that increased activity of IL-1 actually activates keratinocytes before follicular hyperproliferation occurs [13]. So, if it was clear that follicular rupture triggers inflammation in acne, it remains to be elucidated to what extent follicular rupture actually occurs as a consequence of previous inflammation.
HS is a chronic recurrent inflammatory disease, with the origin in the hair follicles of the anatomical zones with apocrine glands. The inflammatory stimulus is the breakage of the hair follicle and the release of keratin and bacteria in the dermis [14]. Hair follicles occlusion with subsequent rupture, destruction of the pilo-sebaceo-apocrine unit and massive secondary inflammation form painful nodules that can progress to superficial abscesses and subcutaneous extension with chronic suppurative sinus tracts and fistula that heal with vicious

scars [15-17]. Other typical HS lesions are open comedones, usually clustered, that communicate with each other, formed by the destruction of the deep component of the pilosebaceous unit $[6,18,19]$. Closed comedones formed by a more superficial damage in the pilosebaceous unit, such as those seen in acne, where are not typically described in HS [20]. Impaired innate immune response also plays a role in HS. Among the evidence of innate immune system impairment are studies that have shown: increased production of IL-1 $\beta$ and (TNF) - $\alpha$ in cell cultures of affected skin [21]; IL-2 level elevated in the serum of HS patients [22]; increased expression of IL-12, IL-17, IL-23 in the skin with HS lesions [23], etc. The pathogenesis of pyoderma gangrenosum is undetermined, although there is different hypothesis about innate and acquired immunity abnormalities [24]. As in acne, suppurative hidradenitis and PASH syndrome, there is evidence of increased IL-1 activity: cutaneous and serum overexpression of IL-1 $\beta$, favorable treatment under anti-IL1- $\beta$ antibody therapy, frequent association with inflammatory systemic diseases [10].

An altered expression of matrix metalloproteinases was observed in each of the three diseases that define PASH syndrome, suggesting a pathogenic role. Mozeika et al obtained elevated levels of metalloproteinase-2 (MMP-2) in keratinocytes, dermal cells, hair follicles units and sinus tracts in HS [25]. In PG patients, overexpression of MMP-2, MMP-9, TIMP- 1 and TIMP-2 was revealed [26]. Production of MMPs by keratinocytes in acne is also involved in hyperkeratinization of the pilosebaceous unit [27].

\section{Genetic profiling and inflammation}

Recent studies have concluded that the genetic basis in PASH syndrome is heterogeneous and is yet to be elucidated [3]. Even though mutations in genes such as PSTPIP1 (pro-lineserinethreonine-phosphatase interacting protein 1) and NCSTN (Nicastrin) have been described so far $[3,28,29]$ the PSTPIP1 mutations can be absent [1].This entity shares common genetic alterations with similar autoinflammatory syndromes, such as PSTPIP1 mutations in PAPA and PAPASH syndrome [3,30,31]. Mutations in PSTPIP1 gene determine the activation of an inflammatory pathway, which results in the activation of IL-1 $\beta$ from its pro-IL-1 $\beta$. Therefore, an exaggerated production of pro 
inflammatory cytokines is observed (especially IL-17), which will lead to neutrophils involvement in the pathogenesis of the disease $[1,2,4,32]$. No circulating autoantibodies nor auto-reactive $\mathrm{T}$ lymphocytes are involved [1].

The genetic abnormalities of the $\gamma$-synthetase complex are involved in epidermal, follicular and cyst defects [33]. Since 1985 Fitzsimmons suggested a possible autosomal dominant transmission in hidradenitis suppurativa, supported by the identification of positive history in first degree relatives of patients in $34.4 \%$ of cases [34]. In $5 \%$ of the patients there were identified different mutations of the three genes that produce component proteins of gamma secretase complex: presenilin-1 (PSEN1), presenilin enhancer-2 (encoded by PSENEN), nicastrin (encoded by NCSTN), and anterior pharynx-defective 1[35]. Reduced gammasecretase activity reduces Notch signaling, involved in normal epidermal and hair follicle differentiation and in modulating T-cell-mediated immune responses [6,36].

\section{Conclusion}

Autoinflammatory diseases are entities described relatively recently, being associated with inflammatory neutrophilic infiltrate (including cutaneous infiltration), and genetically characterized. In the light of these new innate inflammatory disorders, it is a challenge to understand that many of the older diseases can be reclassified into the spectrum of innate immune disorders. Neutrophilic dermatoses were classified primarily based on clinical and histopathological aspects, while autoinflammatory diseases were genetically and pathophysiologically characterized, so that there may be a degree of overlap that could be difficult to clarify.

PASH syndrome may represent a paradigm for gaining new insights into three important diseases - neutrophil mediated cutaneous illnesses: acne, suppurative hidradenitis, pyoderma gangrenosum. The classification of neutrophilic and autoinflammatory skin diseases should be constantly rethought as knowledge of the pathophysiological mechanisms of these conditions develops. Molecular mechanisms involved in monogenic autoinflammatory diseases may play a role in multifactorial neutrophilic diseases.

\section{Conflicts of Interest}

The authors have no conflicts of interest to declare.

\section{Acknowledgements}

All the authors had equal contributions.

\section{References}

1. Niv D, Ramirez J, Fivenson D (2017) “Pyoderma gangrenosum, acne, and hidradenitis suppurativa (PASH) syndrome with recurrent vasculitis." JAAD Case Reports 3(1): 70-73

2. Braun-Falco M, Kovnerystyy O, Lohse P, Ruzicka T (2012) "Pyoderma gangrenosum, acne and suppurative hidradenitis (PASH) - a new autoinflammatory syndrome distinct form PAPA syndrome." J Am Acad Dermatol 66(3): 409-415.

3. Sonbol H, Duchatelet S, Miskinyte S, Bonsang B, Hovnanian A et al. (2018) "PASH syndrome (pyoderma gangrenosum, acne and hidradenitis suppurativa): a disese with genetic heterogeneity." Br J Dermatol 178(1): e17-e18.

4. Marzano, Ceccherini I, Gattorno M, Daniele F, Francesco C, et al. (2014) "Association of Pyoderma Gangrenosum, Acne, and Suppurative Hidradenitis (PASH) Shares Genetic and Cytokine Profiles with Other Autoinflammatory Diseases." Medicine 93(27): e187.

5. Patel F, Fitzmaurice S, Duong C, Young H, Jonathan F, et al. (2015) "Effective strategies for the management of pyoderma gangrenosum: a comprehensive review." Acta Derm Venereol 95(5): 525-531.

6. Griffiths C, Barker J, Bleiker T, Chalmers R, Creamer D (2016) Rook's Textbook of Dermatology. ( $9^{\text {th }}$ edn) Wiley-Blackwell.

7. Dessinioti C, Katsambas A (2013) "Difficult and rare forms of acne." Clin Dermatol 35(2): 138-146.

8. Dhaou B, Boussema F, Aydi Z, Baili L, Rokbani L (2013) "Hidradenitis suppurativa (Verneuil's disease)." Journal of the Saudi Society of Dermatology \& Dermatologic Surgery 17(1): 1-5.

9. Gasparic J, Theut RP, Jemec G (2017) "Recognizing syndromic hidradenitis suppurativa: a review of the literature," J Eur Acad Dermatol Venereol 31: 1809-1816.

10. Navarini, Satoh T, French L (2016) "Neutrophilic dermatoses and autoinflammatory diseases with skin involvement - innate immune disorders." Semin Immunopathol 38: 45-56.

11. Bolognia J, Schaffer J, Cerroni L (2018) Dermatology. ( $4^{\text {th }}$ edn). Elsevier.

12. Zoboulis CC (2001) "Is acne vulgaris a genuine inflammatory disease?." Dermatology 203(4): 277-279.

13. Jeremy, Holland D, Roberts S, Thomson K, Cunliffe W (2003) "Inflammatory events are involved in acne lesion initiation." J Invest Dermatol 121(1): 20-27.

14. Mortimer P, Lunniss P (2000) “Hidradenitis suppurativa.” J R Soc Med 93: 420-422.

15. Kurzen H, Kurokawa I, Jemec G (2008) "What causes hidradernitis suppurativa?" Exp Dermatol 17: 455-472.

16. Ingram J (2018) "Hidradenitis suppurativa: Pathogenesis, clinical features, and diagnosis," Uptodate.

17. Laffert MV, Stadie V, Wohlrab J, Marsch W (2011) "Hidradenitis suppurativa/acne inversa: bilocated epithelial hyperplasia with different sequelae." Br J Dermatol 164(2): 367-371.

18. Habif T (2004)" Clinical dermatology: a color guide to diagnosis and therapy" Mosby.

19. Kamp S, Fiehn A, Stenderup K, Rosada C, Pakkenberg B, et al. "Hidradenitis suppurativa: A disease of the absent sebaceous gland? Sebaceous gland number and volume are significantly reduced in uninvolved hair follicles from patients with hidradenitis suppurativa." Br J Derrmatol 164(5): 1017-1022.

20. Goldsmith L, Katz S, Gilchrest B (2012)" Fitzpatrick's Dermatology in General Medicine", McGraw-Hill.

21. Van der Zee H, Ruiter de L, Broecke VDD (2011) "Elevated levels of tumour necrosis factor (TNF)- $\alpha$, interleukin (IL)-1 $\beta$ and IL-10 in hidradenitis suppurativa skin: a rationale for targeting TNF- $\alpha$ and IL13," Br. J Dermatol 164: 1292-1298.

22. Matusiak Ł, Bieniek A, Szepietowski J (2011) "Soluble interleukin-2 serum level is a useful marker of hidradenitis suppurativa clinical staginig," Biomarkers 14(6): 432-437. 
23. Schlapbach C, Hänni T, Yawalkar N, RE H (2011) "Expression of the IL23/Th17 pathway in lesions of hidradenitis suppurativa." J Am Acad Dermatol 65(4): 790-798.

24. Powell F, Su W, Perry H (1996) "Pyoderma gangrenosum: classification and management." J Am Acad Dermatol 34(3): 395-409.

25. Mozeika E, Pilmane M, Nurnberg B, Jemec G (2013) “Tumour necrosis factor-alpha and matrix metalloproteinase- 2 are expressed strongly in hidradenitis suppurativa." Acta Derm Venereol 93(3): 301-304.

26. Marzano, Fanoni D, Antiga E (2014) “Expression of cytokines, chemokines and other effector molecules in two prototypic autoinflammatory skin diseases, pyoderma gangrenosum and Sweet's syndrome." Clin Exp Immunol 178(1): 48-56.

27. Dreno (2017) "What is new in the pathophysiology of acne, an overview," J Eur Acad Dermatol Venere 31 Supple 5: 8-12.

28. Duchatelet S, Miskinyte S, Join-Labert (2015) "First nicastrin mutation in PASH (pyoderma gangrenosum, acne and suppurative hidradenitis) syndrome." Br J Dermatol 173(2): 610-612.

29. Calderon-Castrat X, Bancalari-DD, Roman-CC, Romo AM, Amorós-CD, et al. (2016) "PSTPIP1 gene mutation in a pyoderma gangrenosum, acne and suppurative hidradenitis (PASH) syndrome." Br J Dermatol 175 194-198.
30. Marzano, Trevisan V, Gattorno M (2012) "Pyogenic arthritis, pyoderma gangrenosum, acne, and hidradenitis suppurativa (PAPASH): a new autoinflammatory syndrome associated with a novel mutation of the PSTPIP1 gene." JAMA Dermatol 149(6): 762-764.

31. Smith E, Allantaz F, Bennet L, Dongping Z, Xiaochong G, et al. (2010) "Clinical, molecular, and genetic characteristics of PAPA syndrome: A review." Curr Genomics 11(7): 519-527.

32. Marzano, Borghi A, Meroni P, M Cugno (2016) “Pyoderma gangrenosum and its syndromic forms: evidence for a link with autoinflammation." $\mathrm{Br}$ J Dermatol 175: 882-891.

33. Pan Y, Lin M, Tian X, Hui-TC, Thomas G (2004) "Gamma-secertase functions through Notch signaling to maintain skin appendages but is not required for their patterning or initial morphogenesis." Dev Cell 7(5): 731-743.

34. Fitzsimmons J, Guilbert P (1985) "A family study of hidradenitis suppurativa." J med Genet 22(5): 367-373.

35. Pink, Simpson M, Desai N, Trembath R, Barker J (2013) " $\gamma$-secretase mutations in hidradenitis suppurativa: New insights into disease pathogenesis." Journal of Investigative Dermatology 133(3): 601-607.

36. Radtke F, Fasnacht N, MacDonald H (2010) "Notch signaling in the immune system." Immunity 32(1): 14-27. 